\title{
As comunidades virtuais como instrumento de educação corporativa: estudo de caso no Tribunal de Contas da União
}

\author{
Emmily Flügel Mathias
}

Universidade de Brasília (UnB)

Gilberto Lacerda Santos

Universidade de Brasília (UnB)

O uso de comunidades virtuais como instrumentos de educação corporativa ainda não se constitui em uma prática comum nas organizações. Apesar do grande número de comunidades disponíveis e das possibilidades de comunicação, interação, troca de conhecimentos e informações que as comunidades virtuais possibilitam, são poucas as situações em que se verifica a sua utilização em situações de educação em meios organizacionais. O presente artigo, decorrente da Tese de Doutorado de Mathias (2010), trata da importância das comunidades virtuais na educação corporativa diante de uma realidade determinada pela sociedade informacional. Assim, parte dos resultados de pesquisa sobre as relações entre educação corporativa, gestão do conhecimento e novas tecnologias da informação, comunicação e expressão, realizada no Tribunal de Contas da União em 2010. A percepção dos participantes da pesquisa e as teorias que discutem questões relacionadas à interatividade e aos processos comunicacionais demonstram que o uso das comunidades virtuais pode representar um importante avanço para a educação corporativa nas organizações.

Palavras-chave: educação corporativa, educação a distância, tecnologia da informação, gestão do conhecimento, sociedade da informação

Artigo recebido em março de 2014. Versão final em julho de 2014. 


\section{Comunidades virtuales como herramientas para educación corporativa: un estudio de caso en el Tribunal de Cuentas de la Unión (Brasil)}

El uso de comunidades virtuales como instrumento de educación corporativa todavía no constituye una práctica común en las organizaciones. A pesar del gran número de comunidades virtuales y de posibilidades de comunicación, interacción y de intercambio de conocimientos e información por meio de ellas, son pocas las situaciones en que hay su uso en situaciones de educación en medios corporativos. Este artículo, basado en la tesis de doctorado de Mathias (2010), trata sobre la importancia de las comunidades virtuales en educación corporativa en el contexto de la sociedad de la información. Por lo tanto, parte de los resultados de la investigación sobre la relación entre educación corporativa, gestión del conocimiento y tecnologías digitales de la información, comunicación y expresión realizada en el Tribunal de Cuentas de Brasil en 2010. La percepción de los participantes de la investigación y las teorías que tratan sobre temas relacionados con los procesos de interactividad y comunicación demuestran que el uso de comunidades virtuales puede representar un avance importante para la educación corporativa en las organizaciones.

Palabras clave: educación corporativa, educación a distancia, tecnologías digitales de la información, gestión del conocimiento, sociedad de la información

\section{Virtual communities as tools for corporative education: a case study in the Brazilian Audit Court}

The use of virtual communities as tools for corporative education is not yet a common practice in organizations, despite the large number of available communities and the possibilities of communication, interaction and exchange of knowledge through them. This article, based on Mathias (2010) doctoral thesis, discusses the importance of virtual communities in corporative education in the context of the Informational Society. The text presents some results from a study about links between Corporative Education, Knowledge Management and New Technologies of Information, Communication and Expression, held at the Brazilian Audit Court, in 2010. The perception of participants and theories that discuss issues related to interactivity and communication processes demonstrates that the use of virtual communities may represent an important advance for corporative education in all organizations.

Keywords: corporative education, distance learning, technologies of information, knowledge management, informational society 


\section{Introdução}

A educação, vista como um importante condutor da capacidade adaptativa das organizações, opera por meio do desenvolvimento de competências capazes de levar os profissionais a se tornarem cada vez mais aptos a enfrentar os inúmeros desafios, em especial aqueles decorrentes das novas configurações econômicas, sociais e profissionais, resultantes de um novo modelo de sociedade, designado por Castells (2000) como sociedade informacional.

A progressiva redução das distâncias entre os indivíduos, e entre esses e o conhecimento, decorre da inserção, na vida cotidiana e no trabalho, das Tecnologias Digitais de Informação, Comunicação e Expressão (TDICE), termo proposto por Lacerda Santos (2010, 2011), para justificar a existência de tecnologias que ampliam cada vez mais a interação entre pessoas, grupos, organizações e até mesmo sociedades.

Entre elas, destacam-se as comunidades virtuais, ambientes criados para que indivíduos possam comunicar-se e trocar impressões, informações e ideias sobre assuntos específicos. Esse tipo de ambiente pode ser visto de forma mais ampla do que um simples espaço de convivência, assumindo contornos típicos de um ambiente de aprendizagem, desde que a ele sejam acrescidos objetivos educacionais claros, bem como seja garantida uma seletividade de conteúdos, vinculada aos interesses organizacionais, por meio de ações de gestão da relação educativa informal que assim toma forma, conforme explicitam Fernandes e Cirillo (2013).

Já é possível identificar algumas experiências relacionadas à criação desse tipo de comunidade, com fins educacionais, nos ambientes corporativos. São comunidades virtuais, em geral, organizadas a partir de blogs, sites ou plataformas também utilizadas para hospedar cursos a distância. Permitem uma colaboração on-line ou mesmo o registro das participações dos usuários, que podem emitir opiniões, criticar, sugerir alternativas, acrescentar informações ou apenas acessálas. Todo esse conteúdo pode servir para enriquecer o trabalho ou ser utilizado como uma ferramenta de aprendizagem para os envolvidos, como apontam Sarruf e Silva (2012), abordando as comunidades de prática virtuais e a troca e criação de conhecimentos em micro e pequenas empresas.

Nesse tipo de comunidade, é possível ensinar e aprender sem, necessariamente, a presença de um professor para conduzir o ritmo, o volume e a qualidade da aprendizagem. Esses atributos decorrem do envolvimento dos participantes, da necessidade de aprendizagem que percebem ao terem contato com o conteúdo ou com os demais participantes, assim como do interesse pelo assunto. 
No entanto, a promoção da educação nas organizações ainda está vinculada a espaços de aprendizagem preestabelecidos, tais como salas de aula ou auditórios, na modalidade presencial, ou mesmo turmas virtuais, na modalidade a distância. A adoção de estratégias decorrentes das TDICE ainda é vista com certa desconfiança, em especial porque existe uma percepção de que esses ambientes existem para que os indivíduos possam fazer amigos, trocar fotos, conversar, enfim, interagir sobre aspectos que não pertencem ao ambiente profissional ou educacional.

Justamente em função da existência de inúmeros casos de comunidades virtuais nas organizações, destinadas a objetivos de natureza diferenciada, é que se propõe a seguinte questão neste estudo: seriam as comunidades virtuais capazes de colaborar efetivamente com a educação corporativa?

Ao estudar a criação de comunidades virtuais no Tribunal de Contas da União (TCU), instituição pública responsável pela fiscalização do uso do dinheiro público no Brasil, foi possível perceber que se trata de uma ação inovadora em termos de educação corporativa, mas que ainda enfrenta resistências por parte dos profissionais que fazem parte do que Prenski (2001) chamou de "imigrantes digitais", ou seja, uma geração que nasceu antes do advento da internet e teve que se adaptar às mudanças por ela provocadas. Apesar de fazerem uso das facilidades promovidas pela internet e pelas TDICE, trata-se de uma geração que ainda apresenta algum tipo de resistência ao seu uso intensivo, inclusive em situações de aprendizagem, conforme pôde ser constatado no estudo realizado no TCU. Possivelmente, em função disso, as experiências-piloto dessa instituição têm sido desenvolvidas em unidades formadas por profissionais oriundos de uma geração mais jovem, portanto, mais habituada ao convívio com as Novas Tecnologias da Informação, Comunicação e Expressão (NTICEs) nos mais diferentes aspectos da vida cotidiana.

O presente artigo foi elaborado a partir dos resultados da pesquisa realizada por Mathias (2010), que investigou, em caráter exploratório, a gestão do conhecimento, a partir do uso das TDICEs, na educação corporativa de uma organização pública, o Tribunal de Contas da União. O método empregado na investigação foi o estudo de caso. Trata-se de uma pesquisa de caráter qualitativo que, na visão de Günter (2006), eleva a compreensão como princípio do conhecimento, estudando relações complexas, em vez de explicá-las apenas por meio do isolamento de variáveis. Foram utilizados três instrumentos de coleta de dados: (a) coleta de dados secundários nas bases de dados do TCU; (b) entrevistas semiestruturadas com dirigentes; e (c) coleta de dados primários por meio de um 
blog. Os resultados foram lançados na base de dados do software Hyper Research - Qualitative Analysis Tool - versão 2.7, para serem processados e analisados a partir das categorias previamente definidas.

\section{Desafios da educação corporativa na sociedade informacional}

Sociedade informacional é um conceito que retrata a sociedade presente pela sua capacidade de uso da informação, disponível nos ambientes digitais e virtuais, para alavancar os processos de aquisição, criação, disponibilização e compartilhamento do conhecimento, por meio de processos comunicacionais que se revertem em inovação e desenvolvimento. A sociedade é informacional porque faz uso da informação de maneira dinâmica, não apenas como elemento de troca, mas de construção de novos caminhos, fazendo com que a economia não seja mais baseada apenas em bens materiais e capital monetário, mas também no conhecimento que as pessoas dispõem (Valente, 2007; Borges, Abreu, Valente, Baranauskas e Bonacin, 2010).

A sociedade informacional trouxe novos desafios ao mundo do trabalho. Segundo Vargas (2003), a demanda por mais aprendizagem, os avanços tecnológicos e a disseminação da informação em larga escala mudaram as perspectivas da educação nas organizações, fazendo com que o modelo tradicional de ensino, que prevaleceu durante séculos, começasse a ser repensado. O ensino tradicional das salas de aula, assim como os demais processos de formação do cidadão e do trabalhador foram estruturados, no passado, de acordo com a lógica da sociedade industrial moderna, que preconizava a especialização do trabalhador para o exercício de cargos específicos com tarefas delimitadas. Tal situação, que caracteriza o modelo de treinamento e desenvolvimento de pessoal, começou a ser modificada quando as organizações passaram a tomar decisões com base em definições estratégicas, considerando não apenas as demandas por produtividade, mas a dinâmica externa determinada pelo mercado, influenciada pela globalização e pelas mudanças de paradigmas típicas da sociedade informacional. Nessa perspectiva, insere-se o modelo de educação corporativa, cuja dinâmica inclui a promoção de ações de aprendizagem intrinsecamente vinculadas à estratégia da organização, promovidas pelas mais diferentes modalidades de ensinoaprendizagem, que ampliam as possibilidades de acesso ao conhecimento. A Figura 1 representa um comparativo da relação existente entre os componentes dos dois modelos citados. 


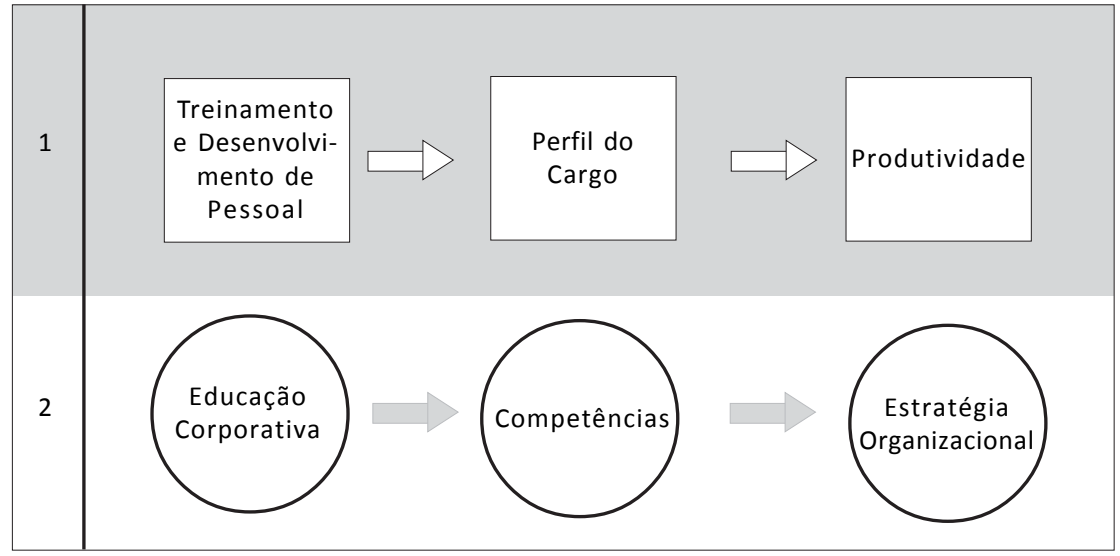

Fonte: MATHIAS, 2010.

\section{Figura 1: Relação entre Treinamento e Desenvolvimento de Pessoal e Educação}

\section{Corporativa}

Insere-se nesse contexto, como mais uma das consequências decorrentes das mudanças promovidas pela sociedade informacional, a gestão do conhecimento, representada pela sua criação, codificação e compartilhamento. O conhecimento, produzido interna ou externamente, tem sido uma das formas mais seguras utilizadas pelas organizações para a construção e ampliação dos seus espaços, incluindo nessa perspectiva a revisão dos paradigmas e das formas de introdução de estratégias de mudança planejada. Para Lundvall e Johnson (1994), o conhecimento é o recurso mais fundamental para a competitividade na moderna economia mundial.

A efetividade da gestão do conhecimento depende, em grande parte, das tecnologias da informação que atuam como facilitadoras do processo comunicacional e, ao mesmo tempo, como mecanismos capazes de agregar novas capacidades à inteligência humana. É pelo surgimento de novas formas de comunicação entre os indivíduos, a transmissão de dados e comandos entre unidades e organizações, ou as reuniões a distância que se retrata o avanço da realidade virtual, que ocupa cada vez mais espaços, até então restritos à presença física, no mesmo lugar e no mesmo tempo, de indivíduos mais qualificados. 0 ensino, as conferências, as consultorias, consultas médicas e até mesmo cirurgias são exemplos de situações em que as novas tecnologias da informação, comunicação e expressão podem modificar a lógica do trabalho e das relações e constituem-se em mecanismos capazes de suportar o aumento da produção e do imenso fluxo de informações que caracteriza a sociedade informacional. 
São, portanto, as TDICEs as principais responsáveis pela ampliação do acesso à informação e ao conhecimento e elementos essenciais para a adaptação das organizações a essa nova realidade. No contexto deste estudo, as TDICEs representam um conjunto de instrumentos e mecanismos digitais, eletrônicos e virtuais que possibilitam a troca de mensagens e informações, a interação social e o posicionamento dos indivíduos diante de situações, fatos ou pessoas com os quais se relacionam, independentemente de tempo ou do lugar onde se encontram. Esse construto foi elaborado a partir da combinação dos conceitos abaixo:

a) informação - refere-se a um fenômeno humano que envolve indivíduos transmitindo e recebendo mensagens no contexto de suas ações possíveis; envolve a interpretação de significados, isto é, a seleção entre as possibilidades semânticas e pragmáticas da mensagem, na perspectiva do receptor;

b) comunicação - trata da interação social por meio de mensagens ou processo pelo qual as relações humanas se realizam; e

c) expressão - pode ser definida como a capacidade do indivíduo de apresentar-se ao mundo, demonstrando seus sentimentos e posicionamentos diante de fatos ou pessoas.

A base para que indivíduos e organizações mantenham-se constantemente atualizados é construída por um processo de aprendizado contínuo, com ênfase no caráter interativo e localizado do aprendizado e da inovação potencializados pelas TDICEs, que ampliam as possibilidades de interconexões entre diferentes indivíduos. Por meio das TDICEs, é possível utilizar as informações disponíveis para agilizar processos de tomada de decisão, incorporar mudanças, bem como promover o aprofundamento no processo de geração de conhecimentos, o que já começa a se tornar um padrão competitivo genérico.

Observa-se que o uso das TDICEs na educação corporativa - em especial nas práticas de educação a distância (EaD); na disseminação das melhores práticas e das "lições aprendidas", facilitadas pelo uso de redes sociais, blogs, bases de pesquisa e outros; no intercâmbio de informações e conhecimento, disponíveis nos portais corporativos, catalisadores de inovações e geração de novos conhecimentos; na criação de comunidades virtuais, facilitadas pelas redes de computadores, nas quais indivíduos e grupos colaboram voluntariamente em função da satisfação de interesses ou solução de problemas - tem facilitado e ampliado as possibilidades de alcance das ações de aprendizagem e da gestão do conhecimento nas organizações, como relata Wenger (2001) e como acontece, por exemplo, no Serviço Federal de Processamento de Dados (Serpro), onde essa estratégia vem sendo explorada com sucesso, conforme relata Lima (2004). 
Em um contexto cuja velocidade e complexidade não permitem mais que se espere pelo melhor momento para se capacitar os funcionários, a educação corporativa, mediada pelas TDICEs, surge como um meio para instrumentalizar a mudança, direcionando o foco para resultados organizacionais mais efetivos, por meio da reunião estratégica da aprendizagem e da gestão do conhecimento. Dessa forma, novas maneiras de se conduzir as ações de aprendizagem têm sido testadas, entre elas, a introdução, cada vez mais frequente, de ações realizadas na modalidade a distância (MathiAs, 2010).

A abrangência da educação corporativa, quando mediada por TDICE, é imensamente aumentada quando as barreiras do tempo e do espaço são ultrapassadas, tendo em vista as inúmeras possibilidades de comunicação oferecidas, a flexibilidade para a participação nas atividades propostas, a inexistência de um local físico onde todos devem dirigir-se em um mesmo horário, a redução dos custos de deslocamento para participar de situações de formação, entre outros, conforme apontam Lastres e Albagli (1999) e Vargas (2003).

Para esses mesmos pesquisadores, outros benefícios podem ser facilmente percebidos nessa relação, além da economia de tempo, redução de custos, maior flexibilidade e ampliação de acesso ao conhecimento. A conversão dos mais variados tipos de informação (incluindo conhecimentos já codificados) para formatos digitais - até mesmo algumas tentativas de reprodução de experiências humanas, como a interação com sons e imagens - permite que tais informações sejam registradas, manipuladas e reproduzidas por máquinas digitais a qualquer momento. É o que Lastres e Albagli (1999) chamam de "vantagem digital", isto é, uma tradução universal que poupa recursos, é fácil de armazenar, transportar, copiar, medir e manipular.

O processamento, armazenamento, recuperação e comunicação da informação, em qualquer formato, sem interferência de fatores como a distância, o tempo ou o volume, representam uma revolução no modo de se trabalhar e viver junto. $\mathrm{Na}$ visão de De Luca (2003), essa condição corresponde ao modelo construtivista sociointeracionista de educação, no qual a aprendizagem se dá de forma autônoma e colaborativa, isto é, por meio da comunicação e da colaboração que ocorre nos ambientes virtuais de aprendizagem (AVA).

São os AVAs que permitem e contribuem para o armazenamento e reutilização do conhecimento, na medida em que possibilitam que o processo de aprendizagem envolva mais de um aprendiz e os registros que ocorrem durante as trocas fiquem disponíveis a todos. Isso implica o desenvolvimento de um forte elo entre a teoria e a prática, uma vez que não se tem apenas o registro do conhecimento puro, mas as interpretações do mundo real e das aplicações daquele conhecimento (LACERDA SANTOS, 2011). 


\section{Comunidades virtuais}

As TDICEs, como mediadoras dos processos de educação corporativa e, ao mesmo tempo, como instrumentos de aproximação entre os profissionais e os conhecimentos disponíveis, têm exercido um papel essencial relacionando a aprendizagem à gestão do conhecimento. Segundo Castells (2000), tais tecnologias representam um novo paradigma que expressa a essência das transformações tecnológicas e das suas relações com a economia e com a sociedade, cujas características fundamentais ele assim descreve:

a) A informação é a matéria-prima, e o desenvolvimento das tecnologias ocorre para que o homem possa atuar sobre ela e não o contrário.

b) Todas as atividades humanas tendem a ser afetadas diretamente pela tecnologia, porque a informação é parte integrante de toda atividade humana, individual ou coletiva.

c) Há um predomínio da lógica de redes em qualquer tipo de processo.

d) A flexibilidade configura uma das características da tecnologia, pois essa favorece processos reversíveis, permite modificação por reorganização de componentes e tem alta capacidade de reconfiguração.

e) Há uma crescente convergência de tecnologias, principalmente da microeletrônica, telecomunicações, optoeletrônica, computadores e da biologia, interligando as áreas e transformando-as em categorias de análise de todos os processos.

Para Werthein (2000), de todas as características do paradigma da tecnologia da informação, a flexibilidade é a que mais se relaciona com o conceito de aprendizagem. A capacidade de reconfiguração do sistema permite a incorporação da mudança, tanto no âmbito das organizações quanto na capacidade de adaptação dos trabalhadores, consumidores, usuários e produtores. Para o autor, isso coloca a questão da formação continuada como um requisito da sociedade informacional. A revolução da educação, promovida pela disponibilidade de recursos que a tecnologia da informação fornece, sofreu um maior impulso quando as novas possibilidades de comunicação começaram a ser disponibilizadas.

Nesse contexto, as comunidades virtuais possibilitam o acesso ao conhecimento, assim como a colaboração em torno de determinados temas. Embora as TDICEs sejam meios de acesso ao conhecimento, o seu uso adequado, isto é, a capacidade de escolha acertada desses meios em função dos objetivos educacionais pretendidos pode influenciar os resultados de aprendizagem pretendidos e, pela lógica, a melhoria do desempenho organizacional. O próprio ciberespaço pode ser considerado um ambiente virtual de aprendizagem, dada a sua natureza aberta e 
flexível. Com isso, os conceitos de colaboração, cooperação e interatividade passam a ser contemplados nas comunidades virtuais (MATHIAS, 2010).

Ao estimular a criação de comunidades virtuais, o TCU inova ao adotar mecanismos típicos de uma abordagem sociointeracionista, utilizando as TDICEs como recursos de aprendizagem. Essa abordagem tem como fundamento os estudos desenvolvidos por Vygotsky, Wallon, Piaget e outros, segundo os quais, o indivíduo aprende pela interação com o outro. Faz uso de artefatos, como a tecnologia, fruto da evolução da sociedade, que possibilita o confronto entre o conhecimento de um e de outro, promovendo o seu desenvolvimento cognitivo. É nessa perspectiva que se criam as redes de cooperação entre indivíduos, que permitem a construção coletiva do conhecimento, base dos processos de aprendizagem das comunidades virtuais do tribunal. Trata-se de uma abordagem normalmente identificada em ambientes de educação formal e que consiste em um movimento inovador quando se trata de situações de educação corporativa, como a abordada neste texto.

Para a criação dessas comunidades virtuais, o tribunal incentiva o uso dos recursos do Ambiente Virtual de Educação Corporativa do TCU (AVEC-TCU), em especial os fóruns, cujo conteúdo fica armazenado e pode ser utilizado na melhoria dos cursos já editados, visto que a qualidade e a variedade das participações são significativas. Trata-se de uma estratégia de explicitação de temas por meio de fóruns de discussão temáticos, temas esses que serão empregados na melhoria dos cursos de formação continuada ou na criação de novos cursos, os quais podem, por sua vez, alimentar novos fóruns de discussão e novas comunidades virtuais. A efetividade e o impacto dessa estratégia de explicação e de exploração de conhecimentos dos membros da cadeia de valor do TCU são continuamente avaliados, tanto ao final de cada curso quanto em discussões específicas nos próprios fóruns de discussão.

De acordo com a Secretaria de Obras do TCU, que mantém duas comunidades virtuais, praticamente todos os servidores utilizam o ambiente virtual, especialmente no período entre março e julho, quando ocorre o maior número de auditorias. Essas comunidades virtuais são utilizadas como meio para que os servidores interessados possam fazer perguntas, discutir procedimentos, linhas de raciocínio, debater acerca de posicionamentos divergentes, entre outros. Apesar de parecerem meros instrumentos facilitadores do trabalho, tais comunidades virtuais começaram a ser percebidas como ferramentas de aprendizagem e passaram a representar uma evolução no uso das TDICEs no TCU, assim como uma evolução na perspectiva pedagógica que sustenta a educação corporativa do tribunal, segundo avaliação de $36,14 \%$ dos participantes da investigação aqui relatada. Hoje, por meio 
dessas e de outras comunidades virtuais, tem-se a oportunidade de registrar as participações nos fóruns e de torná-las disponíveis para futuros acessos. Alguns tópicos são discutidos semanalmente, como os relacionados à jurisprudência; por isso, estão sempre atualizados e podem ser acessados quando necessário. As estratégias de moderação são diversas e variam de acordo com o público envolvido nas comunidades virtuais e com os objetivos visados pelos gestores de pessoas e de conhecimento no ambiente do TCU. Nessa perspectiva, determinados temas requerem uma moderação mais contundente e cotidiana, enquanto que outros permitem que os participantes discutam mais livremente, até mesmo sem necessidade de moderadores gerando atividades e propondo temas novos. 0 princípio do conteúdo significativo é o principal agregador de valor às comunidades virtuais em foco, o que tanto atrai novos participantes quanto os fideliza em discussões que são pertinentes e que, claramente, contribuem com a produção ou com a explicitação de conhecimentos úteis para o tribunal.

A pesquisa realizada no ambiente do TCU por Mathias (2010) revela que, em situação de educação corporativa, as pessoas aprendem melhor sozinhas, mas criam conhecimentos em espaços coletivos. Dos respondentes, 55,42\% afirmam haver um intenso compartilhamento de conhecimentos entre as áreas, daí a importância de se estimular a criação de ambientes que não apenas sirvam como mediadores dos processos comunicacionais, mas que possibilitem o armazenamento de informações e conhecimentos importantes para o tribunal e para seus beneficiários. Até mesmo porque $66,27 \%$ dos respondentes afirmam que aprendem mais estudando sozinhos, o que demonstra que a disponibilidade e o acesso aos conteúdos de interesse, por si só, já representam importantes elementos da aprendizagem. Cabe às organizações o desenvolvimento de estratégias capazes de identificar, selecionar e organizar esses espaços; isso porque as possibilidades das NTICEs são inúmeras, assim como os canais de acesso à informação; entretanto, nem sempre o conteúdo disponível interessa a um determinado contexto, tornando essencial a vinculação entre a estratégia organizacional e a gestão do conhecimento.

$\mathrm{Na}$ organização pesquisada, $61,45 \%$ dos servidores afirmam criar conhecimentos e reconhecem que esses são convertidos em normas ou leis, que beneficiam a própria instituição. No entanto, para $22,89 \%$ deles, o compartilhamento do conhecimento ainda é dificultado por razões ligadas ao poder conferido àqueles que o detêm. Entretanto, os dados revelam que isso não ocorre nas ações de aprendizagem, porque, nesses ambientes, 84,34\% dos respondentes veem com bons olhos as trocas e as participações dos colegas. Dessa forma, a criação de espaços onde as pessoas possam, além de trocar ideias e 
informações, produzir e acessar conhecimentos pode representar um estímulo para o estabelecimento de relações cada vez mais colaborativas na organização, em especial se a eles estiverem vinculados propósitos educacionais claros.

\section{Conclusões}

A questão central deste estudo, referente à capacidade das comunidades virtuais colaborarem com a educação corporativa nas organizações, repousa na forma como o aprendizado é visto na sociedade informacional. A amplitude do acesso ao conhecimento, aliada à autonomia da busca e do acesso, subvertem a lógica das aulas programadas e dos temas delimitados, encontrados tanto na modalidade presencial quanto na modalidade a distância dos treinamentos tradicionais. As inúmeras possibilidades de interação e troca de informações proporcionadas pelas TDICEs rompem as barreiras do acesso ao conhecimento e, simultaneamente, desafiam os limites da hierarquia, colocando o indivíduo em confronto com a organização e consigo mesmo.

Se, conforme demonstrou a pesquisa, informação e conhecimento são atributos que conferem poder àqueles que os detêm, as TDICEs tanto podem subverter as antigas estruturas organizacionais quanto terem o seu uso restrito aos limites suportados pela cultura da organização. Nesse sentido, é possível que o poder do conhecimento individualizado seja gradativamente substituído pelo poder do pertencer, adquirido por aqueles que participam intensamente da lógica da sociedade informacional. Como membros de comunidades virtuais e diferentes redes de relacionamento, os indivíduos tornam-se mais visíveis em uma sociedade altamente complexa, onde não existem mais distâncias e onde o individual e o coletivo se confundem. Mais visíveis, esses indivíduos podem assumir papéis de destaque na organização, semelhantes aos fenômenos de audiência e popularidade de artistas, políticos, blogueiros, impulsionados pelos recursos das TDICEs. Tal fenômeno, no contexto das organizações, estimula uma visibilidade interna que, diferentemente das relações hierárquicas tradicionais - em que os participantes são selecionados a partir de critérios específicos -, expõe aqueles que mais fazem uso das TDICEs e torna invisíveis aqueles que as ignoram.

Ao assumirem parte da responsabilidade pela formação dos trabalhadores, as organizações precisam se estruturar para gerenciar a tensão entre as novas competências dos indivíduos, que, com o auxílio das TDICEs, podem adquirir conhecimentos de forma autônoma, e a necessidade de adaptação da sua estrutura, processos, política e, principalmente, da sua cultura em uma sociedade informacional. 
Para sobreviver no contexto da sociedade informacional, a organização precisa enfrentar diversos desafios. Entre eles, ser capaz de promover educação corporativa potencializando o uso das TDICEs, em especial, na criação de espaços colaborativos, como as comunidades virtuais, para que os indivíduos possam colocar em prática seus conhecimentos e agregar valor ao patrimônio de conhecimentos da organização.

\section{Referências bibliográficas}

Borges, M.; Abreu, J; Valente, J. A.; Baranauskas, M. C.; e Bonacin, R. Aprendizagem em empresas na era das tecnologias digitais. In Anais do Congresso Brasileiro de Informática na Educação, João Pessoa (PB), v. 1, p. 126-138, 2010.

Castells, M. A Sociedade em Rede. São Paulo: Paz e Terra, 2000.

DE LUCA, R. Educação a distância: ferramenta sob medida para o ensino corporativo. In: SILVA, M. A. Educação online: teorias, práticas, legislação, formação corporativa. São Paulo, Edições Loyola, 2003.

Fernandes, F. e Cirillo, M. A. Redes Sociais Digitais: apontamentos e reflexões quanto sua interação com a cultura e comunicação organizacional. Anais do V Seminário Internacional de Pesquisas em Comunicação, Universidade Federal de Santa Maria, 2013.

GüNTheR, H. Pesquisa Qualitativa Versus Pesquisa Quantitativa: Esta é a questão? Psicologia: Teoria e Pesquisa, v. 22, n. 2, p. 201-210, maio-ago. 2006.

Lacerda Santos, G. Ensinar e Aprender no Meio Virtual: Rompendo Paradigmas. Educação e Pesquisa, v. 37, n. 2, p. 307-320, 2011.

Formar professores para a educação mediada por tecnologias: elucidação da problemática por meio de seis investigações acadêmicas. In: LACERDA SAntos, Gilberto e AndRADE, Jaqueline Barbosa Ferraz de (org.). Virtualizando a escola: migrações docentes rumo à sala de aula virtual. Brasília: Liber Livro, 2010.

Lastres, H. e Albagl, S. Informação e Globalização na Era do Conhecimento. Rio de Janeiro: Editora Campus, 1999.

LıMA, M. P. Comunidades de Aprendizagem em Meios Organizacionais. Revista Latinoamericana de Tecnologia Educativa, v. 3, n. 2. Acessado em 12/05/2014. Disponível em http://campusvirtual.unex.es/revistas/index.php/relatec/article/ view/163/153.

Lundvall, B. e Johnson, B. Why all this fuss about codified and tacit Knowledge? Industrial and Corporate Change, no 2, p. 245-62, 2003.

Mathias, E. F. Um estudo sobre a relação entre o uso das NTICE na Educação Corporativa e a Gestão do Conhecimento de uma instituição pública: o caso do TCU. Tese de Doutorado. Brasília, Universidade de Brasília, 2010. 
Prensky, M. Do They Really Think Differently? On the Horizon. Vancouver: NCB University Press. v. 9, n. 6, 2001.

SARruf, P. G. e Silva, H. F. N. Comunidades de prática virtuais e a troca e criação de conhecimentos em micro e pequenas empresas. DataGramaZero - Revista de Informação -, v. 13, n. 1, fevereiro de 2012. Acessado em 12/05/2014. Disponível em http://www.dgz.org.br/fev12/Art_02.htm.

VAlente, J. A. A crescente demanda por trabalhadores mais bem qualificados: a capacitação para a aprendizagem continuada ao longo da vida. In: VALENTE, J. A.; Mazzone, J.; Baranauskas, M. C. C. (Eds). Aprendizagem na era das tecnologias digitais. São Paulo: Cortez, 2007.

VARGAS, M. R. M. Educação a distância no contexto da mudança organizacional. In: Lima, S. M. V. (Org.). Mudança organizacional: Teoria e Gestão. Rio de Janeiro, FGV, 2003.

Wenger, E. C.; Snyder, W. M. Comunidades de Prática: a fronteira organizacional. Aprendizagem Organizacional. Harvard Business Review: Editora Campos, 2001. p. 926.

Werthein, J. A sociedade da informação e seus desafios. Ciência e Informação, Brasília, v. 29, n. 2, p. 71-77, 2000.

\section{Emmily Flügel Mathias}

Doutora em Educação pela Universidade de Brasília (UnB), consultora em educação corporativa e diretora da empresa Insight Consultores. Contato: emmily@insight-consultores.com.br

Gilberto Lacerda Santos

Doutor em Sociologia do conhecimento científico e tecnológico pela Universidade de Brasília (UnB) e Ph.D. em Educação pela Universidade Laval (Canadá). É professor associado IV da Faculdade de Educação da Universidade de Brasília. Contato: glacerda@unb.br 